\title{
Noise measurements in optical amplifiers
}

\section{Lionel Jacubowiez, Thierry Avignon, Nicolas Dubreuil, Marc Bondiou}

Lionel Jacubowiez, Thierry Avignon, Nicolas Dubreuil, Marc Bondiou, "Noise measurements in optical amplifiers," Proc. SPIE 9664, Ninth International Topical Meeting on Education and Training in Optics and Photonics, 96642F (24 October 2005); doi: 10.1117/12.2207562

SDIE Event: Ninth International Topical Meeting on Education and Training in Optics and Photonics, 2005, Marseille, France 
Ref ETOP012

\title{
Noise measurements in optical amplifiers
}

\author{
Lionel Jacubowiez, Thierry Avignon, Nicolas Dubreuil and Marc Bondiou
}

\begin{abstract}
We describe an experiment that allows advanced master students in optical science and engineering to understand noise measurements in optical amplifiers (e.g. Erbium Doped Fiber Amplifiers). Noise and signal are studied then measured in both electrical and optical domains. The degradation of the signal to noise ratio through EDFA's along a real or simulated long-haul optical link can also be studied.
\end{abstract}

(C)2005 Optical Society of America

OCIS codes: 060.0060 Fiber optics and optical communications, 060.2320 Fiber optics amplifiers and oscillators, 250.4480 Optical amplifiers, 270.5290 Photon statistics

\section{Introduction}

\section{Summary}

Optical amplifiers are widely used and key devices in optical fiber communications. Our labwork experiment uses a commercial-type EDFA with all suitable devices (tunable source, synthetizer, optical attenuators, high speed/low noise photodiode, electrical and optical spectrum analyzers).

In a first part, students study typical characteristics of an EDFA using an optical spectrum analyzer (OSA): they measure the amplified spontaneous emission (ASE), optical gain, gain saturation with respect to pump level and signal level, non uniformity of the gain with respect to wavelength, etc.

In a second part, the students start studying noise generated by optical detection of different types of optical flux: attenuated laser flux then ASE flux. They find the evolution of noise level measured with an electrical spectrum analyzer to be completely different between the 2 sources.

Then, they measure an RF-range (100 MHz to $1 \mathrm{GHz}$ ) AM signal optically transmitted along a fiber link at different points : at the input of the EDFA then at the output. The degradation of SNR through the EDFA (the so-called "noise figure" NF) is derived then compared to the measurement performed through the optical domain by the OSA using its plugged-in application "optical amplifier test"). The ultimate objective is to obtain close (or at least compatible) measurements by both approaches.

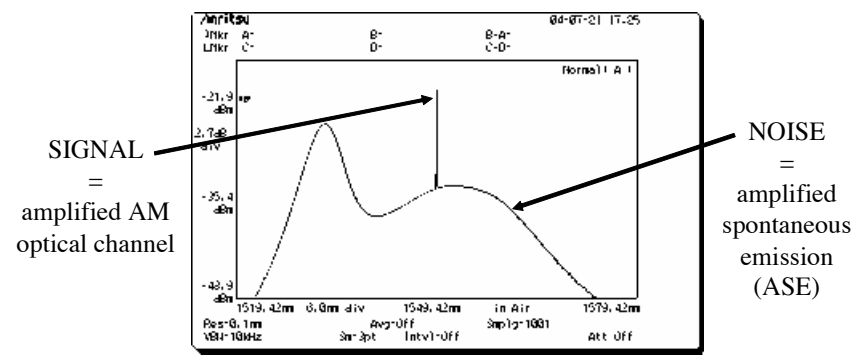

Fig.0 : typical EDFA's output spectrum 


\section{Experiment}

\subsection{Set-up}

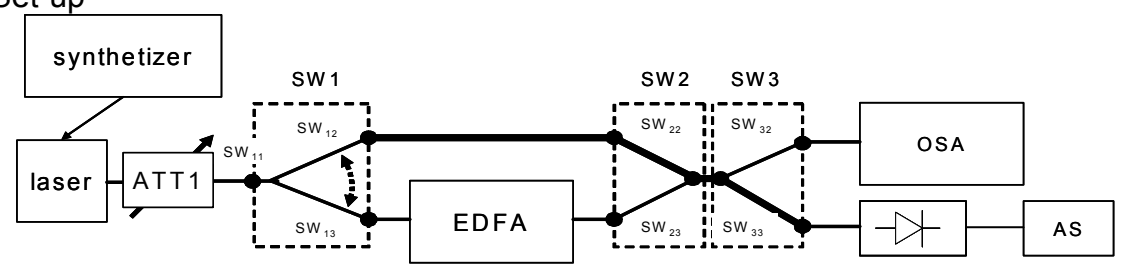

Fig. 1. Experimental set-up.

Fig.1 shows the set-up of the experiment. The emitter is a commercial tunable extended cavity diode laser in the $1500-1600 \mathrm{~nm}$ range which amplitude is modulated by a RF synthetizer. ATT1 is a calibrated optical attenuator. SWk are optical switches and SWij their input or output ports. The receiver is a low noise amplified high-bandwidth photodiode.The optical spectrum analyzer (OSA) is a grating-type standard resolution $(0.1 \mathrm{~nm})$ device and AS is a RF electrical analyzer.

2.2 Noise measurements with laser and ASE sources
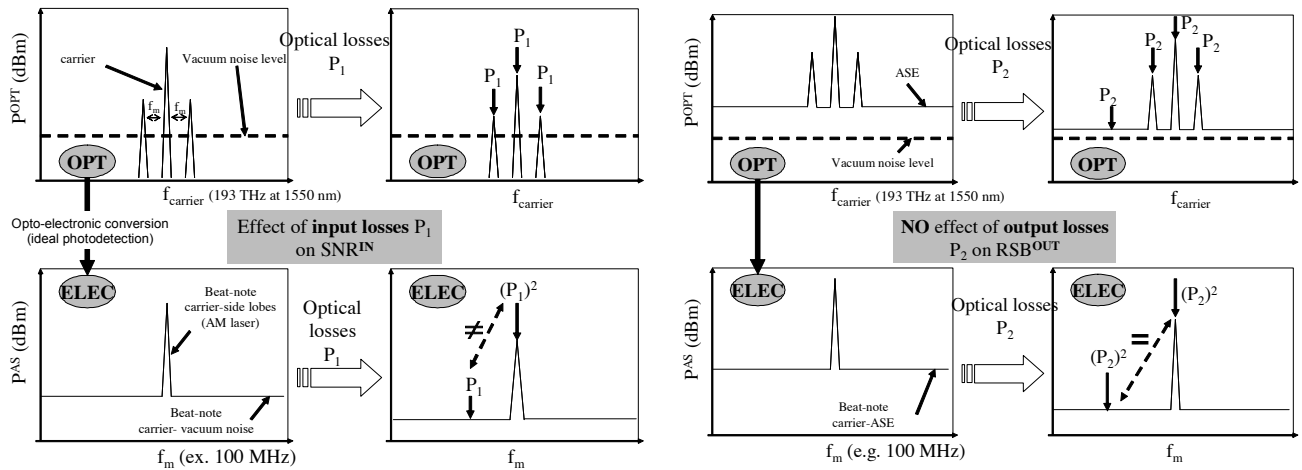

Fig. 2. Effect of input (left) and output (right) losses on SNR (OPT stands for OPTical domain, ELEC for ELECtrical domain)

Fig. 2 summarizes the behavior of the noise of a amplitude modulated laser with respect to the optical attenuation (equivalent to the study of the effect of input losses on the SNR): on top, spectra in the optical domain (OPT) require to represent the "vacuum noise level" which is introduced (and usefull) in quantum optics courses. For the study of the noise itself (generated by the laser), the modulation is not necessary. On right side of fig. 2, the same type of spectra is obtained for the amplified AM laser with respect to the optical attenuation (equivalent to the study of the effect of output losses on the SNR 2.3 SNR degradation (NF) 

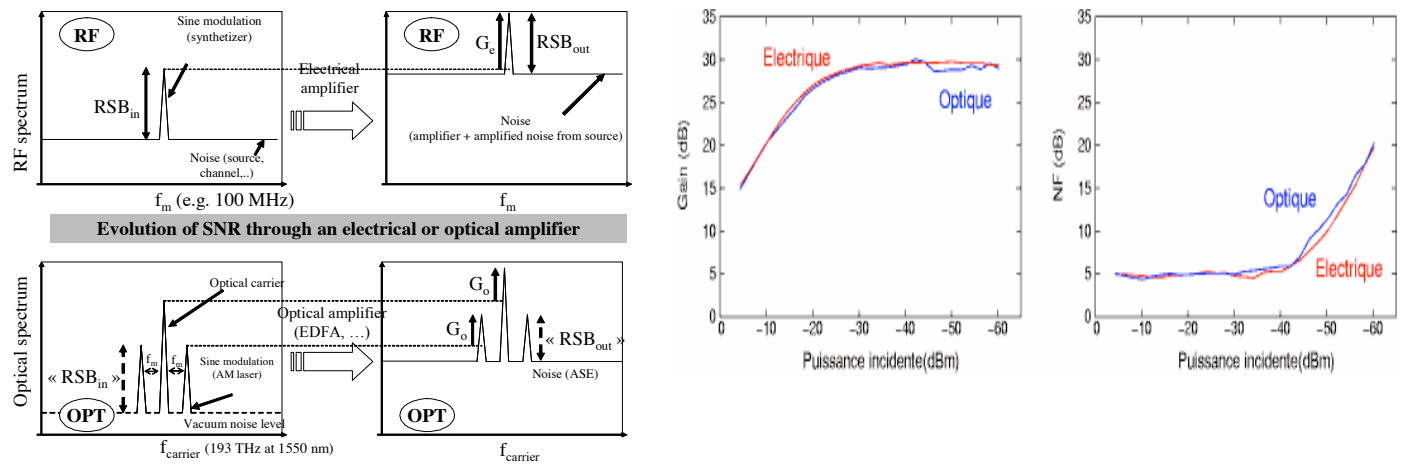

Puissance incidente(dBr)

Puissance incidente(dBm)

Fig. 3 .Definition of SNR and NF through an electrical or optical amplifier (left) : application to the case of an EDFA for both optical and electrical NF measurements methods

The degradation of SNR through (NF) is fairly well verified. The agreement between optical and electrical NF measurements is quite good, depending on each side on 1) the formula used in the OSA application (it can be derived and calculated separately) and 2) the calibration of the photodiode and electrical analyzer.

In conclusion, this labwork is a good opportunity to familiarize students with a key optoelectronic component in optical fiber communications and, in the same time, open to misleading concepts (classical or quantum noise) and measurements techniques. 